\title{
Le fortificazioni litoranee di Terra d'Otranto: una panoramica sulle torri costiere della provincia di Lecce
}

The coastal fortifications of Terra d'Otranto: a panoramic view of the coastal towers in the province of Lecce

\section{Giuseppe Tricarico}

Independent researcher, Terlizzi (BA), Italy, giuseppetric@hotmail.it

\begin{abstract}
During the Modern Age we witnessed the birth, consolidation and decline of great powers that dragged numerous political and religious conflicts with them. The Mediterranean Sea, as area of contact between the most distant Empires, experienced an era of intense naval activity in the form of piracy, race wars and armed deterrence, spreading along its shores with coastal watch towers.

The organization of the defensive coastal system took place in intimate relationship with the territory, strongly characterizing the coasts which for centuries have seen them as the unique anthropical presence. Their exclusively military character has, however, inhibited their reuse over time, arousing until a few decades ago the disinterest of the community and their disavowal of architectures worthy of protection. The knowledge of the historical events and the morphotypological characteristics of the Apulian system of coastal towers thus becomes the starting point for their acknowledgment as fundamental identifying characters of the territory, finalizing their study to the re-appropriation of these assets by the community as strategical vehicles for the transmission of the local history and its intrinsic values. The classification of the towers in the province of Lecce has made them the object of spatial and typological analyses produced with the help of the opensource software "Quantum GIS" and geo-referenced on the official cartographic bases.
\end{abstract}

Keywords: Apulian defensive system of coastal fortifications, Terra d'Otranto, Lecce, GIS classification.

\section{Introduzione}

Come accaduto lungo tutte le coste del Regno di Napoli, anche nella penisola pugliese la costruzione del sistema di torri litoranee di avvistamento non può discorrere da quel grande progetto di stampo imperiale voluto da Filippo II ed ivi realizzato in seguito all'Orden General, impartito dal Vicerè Pedro Afan de Ribera nel 1563 ed affiancato nello stesso anno dalla formazione di una Milizia territoriale, proseguendo nell'opera di fortificazione costiera già avviata in passato per iniziativa privata e sotto i governi normanno, svevo, angioino, aragonese e vicereale di Pedro de Toledo poi, imponendo la Terra d'Otranto quale "importantissimo laboratorio per la sperimentazione di nuove forme dell'architettura militare, da lanciare poi a scala internazionale" (Brunetti, 2006).

Da quel momento, oltre a rafforzare le torri già esistenti e rendere efficienti quelle in cattivo stato, "lungo tutte le coste gli ingegneri regi avrebbero stabilito i luoghi ove costruire altre torri di 
vedetta, ad una distanza l'una dall'altra per cui ciascuna fosse visibile dalle due più vicine. $\mathrm{Ne}$ sarebbe risultata una ininterrotta linea fortificata, i cui elementi potessero comunicare visivamente, tramite segnali fra loro, mediante fumate di giorno e il fuoco di notte, e acusticamente mediante una campana" (De Vita, 2001).

Per avere un ordine di grandezza, alla fine del XVI secolo il solo Regno di Napoli annoverava complessivamente più di 300 torri; nel 1748 erano 379 , delle quali più di un terzo in Puglia e 80 nella sola Terra d'Otranto (Cosi, 1930).

\section{Il carattere sistemico delle torri costiere}

L'efficacia di una torre di avvistamento rimane quasi nulla se non considerata in rapporto all'intero sistema nella quale è inglobata. Esse furono erette esclusivamente allo scopo della difesa militare del litorale per il quale svolsero la funzione di avvistamento e comunicazione del pericolo, che impose la necessità di disporle nella giusta relazione con le città e con le altre fortificazioni, oltre che tra loro e con il territorio. Può infatti affermarsi che «fu il territorio la causa della particolare dislocazione di esse e che il territorio stesso dalla loro installazione ebbe sottolineati e valorizzati i suoi punti focali» (Mutalipassi, 2006). Per questa ragione esse si configurano come un interessante indicatore per conoscere la storia pregressa dell'organizzazione del territorio, facendo di queste architetture uno dei maggiori veicoli per conservare e trasmettere quei significati che costituiscono il carattere ambientale di un luogo e, dunque, la sua identità, e, per questo, punti strategici per la valorizzazione del territorio (Pace, 1992). Travisare il carattere sistemico assunto dalle torri costiere nella storia preclude dunque una conoscenza approfondita della loro origine, delle loro funzioni e dei loro caratteri.

\section{I caratteri tipologici delle torri costiere}

Il periodo di transizione intercorso tra Medioevo ed Età Moderna è stato per le strutture difensive teatro di profonde trasformazioni dovute principalmente all'applicazione in ambito bellico della polvere da sparo e della conseguente mutazione delle tecniche ossidionali. Rispetto all'impianto medievale, caratterizzato dalla prevenzione dell'elemento "sorpresa" mediante la difesa in posizione dominante, le torri furono abbassate ad un unico livello ed inspessite con la realizzazione di muri a scarpa per meglio resistere ai colpi di cannone, nonché preferite dapprima cilindriche e in seguito poligonali con uno spigolo rivolto verso il mare; le feritoie e le merlature vennero allargate per alloggiarvi le nuove armi mentre caditoie e guardiole scomparvero poiché ormai considerate elementi di debolezza, insieme alle componenti lignee, facilmente incendiabili (Petrignani, 2001).

Nonostante queste modificazioni, gran parte delle torri costiere presenta dei caratteri tipologici comuni dettati dalle caratteristiche dei materiali locali impiegati nella loro costruzione, dalle tecniche costruttive tipiche dell'arco cronologico di edificazione e dalle necessità pratiche delle loro funzioni.

Per la loro costruzione sono state impiegate esclusivamente pietre locali, corrispondenti al tufo nella terminazione salentina e, più spesso, alla sua variante concrezionaria più dura e resistente chiamata càrparo (De Giorgi, 1981). Quasi tutte prevedevano l'accesso al primo piano tramite una scala in legno che quando non utilizzata veniva retratta; successivamente, ad alcune di esse vennero aggiunte delle scale esterne in materiale lapideo munite di ponte levatoio o, quando la loro funzione difensiva era ormai declinata, saldate direttamente alla torre. Alcune torri presentano inoltre una scala interna al paramento murario che le collega al piano terreno. Spesso, quasi sempre contenuta nel muro a monte, in questo livello vi era una cisterna che raccoglieva l'acqua del lastrico per l'approvvigionamento idrico dei torrieri alla quale si accedeva da una botola di collegamento con il piano abitabile (De Vita, 2001).

\section{La catalogazione delle torri costiere della provincia di Lecce}

Il presente studio si concentra sulla porzione delle torri costiere pugliesi ricadente nella provincia di Lecce, facente parte, insieme a quelle di Brindisi e Taranto, della più antica provincia di Terra d'Otranto. 
I dati utilizzati nelle analisi sono stati in gran parte estrapolati dal censimento effettuato dall'arch. Vittorio Faglia con la collaborazione di Fernando Bruno, Gabriello Rosso e Arnaldo Manuele (Faglia, 1978), che nei suoi anni di attività si è occupato più in generale dello studio delle torri costiere del Regno di Napoli situate nelle attuali regioni del Meridione comprese tra le Marche e la Campania.

Sono state individuate 61 torri $^{1}$, schedate indicando per ognuna di esse, in forma tabellare, le caratteristiche dimensionali, tipologiche e spaziali affiancandole alle loro rispettive coordinate geografiche, proiettate nel sistema di riferimento WGS84 UTM 33N funzionalmente alla loro georeferenziazione. Il sistema è stato successivamente trasposto su supporto cartografico tramite il software opensource Quantum GIS con il quale sono state elaborate delle analisi grafiche rivelanti, in una vista d'insieme, le caratteristiche tipologiche e spaziali delle singole torri, la loro periodizzazione cronologica e il loro attuale stato di conservazione in un'ottica relazionale.

I risultati ottenuti mettono in luce le diverse relazioni che collegano i manufatti, nel proposito di superare la visione frammentata degli interventi che hanno finora consentito la loro conservazione in maniera solamente parziale $\mathrm{e}$ nell'ottica di una valorizzazione integrata che li consideri come tasselli fondamentali di un sistema che di fatto rappresenta un unicum.

\subsection{Tipologie}

Dall'analisi delle torri costiere della provincia di Lecce emergono cinque tipologie principali²:

\section{a. Torri a pianta quadrata tipiche del Regno ${ }^{3}$}

Costruite prevalentemente nel decennio 1565-75 in seguito all'Orden General di Parafan de Ribera (1563), sono tra le più numerose. Hanno pianta quadrata, basamento scarpato (del $5 \%$ circa) e solitamente tre caditoie in controscarpa (dell' $8 \%$ circa), e la misura del lato esterno varia tra i $10 \mathrm{e}$ i $12 \mathrm{~m}$. Gli spigoli sono sempre costruiti con materiale squadrato mentre le pareti sono più frequentemente realizzate con materiale irregolare. Non sono presenti nell'area tra Otranto e Leuca dove già precedentemente le iniziative locali avevano provveduto a costruirne altre.

\section{b. Torri a pianta quadrata non tipiche del Regno}

Quasi tutte concentrate nel territorio di Nardò, presentano un basamento scarpato separato con un cordolo dal corpo parallelepipedo e un coronamento in leggero sbalzo su beccatelli, dotato di caditoie in analogia con le torri delle masserie fortificate; possiedono una scala interna in spessore di muro e quasi tutte una scala esterna quasi certamente posteriore. La struttura, quasi sempre in conci regolari, è più imponente di quella delle torri vicereali con corpo di lato da 11 a $16 \mathrm{~m} \mathrm{e}$ altezza da 14 a $18 \mathrm{~m}$. La loro dimensione è giustificata dall'influenza ecclesiastica esercitata sul feudo di Nardò e dalla intensa attività agricola praticata in quest' area; alcune di esse sono infatti collegate all'organizzazione delle masserie più interne. Le rimanenti torri quadrate sono ricondotte alla tipologia di quelle delle masserie fortificate o dello Stato della Chiesa.

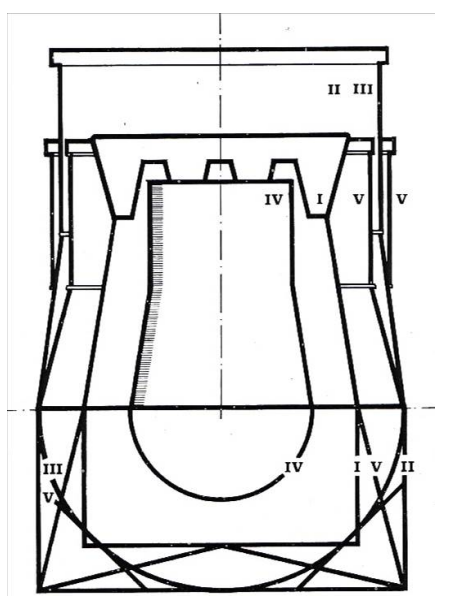

Fig. 1. "Tipologia delle torri costiere di Terra di Otranto e loro rapporto dimensionale. I) Torre a base quadrata tipica del Regno. II) Torre a base quadrata grande (della serie di Nardò), III) Torre a base circolare grande e media. IV) Torre a base circolare piccola (della serie di Otranto), V) Torre a base ottagonale e a cappello da prete" (Faglia, 1978). 


\section{c. Torri a pianta circolare grandi e medie}

Aventi diametro alla base compreso fra 11 e 17 $\mathrm{m}$, sono meno numerose delle precedenti e sono concentrate nella parte meridionale della provincia, quasi sicuramente costruite su iniziativa di enti periferici o privati.

\section{d. Torri a pianta circolare piccole}

Concentrate nella costa alta e scoscesa a sud di Otranto, risultano essere tra le prime costruite, probabilmente in seguito all'eccidio del 14801481 per l'urgenza di difendersi dalle minacce turche. Composte da una base troncononica in pietrame e da un corpo cilindrico con all'interno un unico ambiente voltato, possedevano una dimensione minima per l'alloggiamento di una vedetta, giustificata da necessità di urgenza ed economia: con un diametro alla base inferiore ai $9 \mathrm{~m}$, il loro volume è pari a $1 / 3$ di quello di una torre vicereale e $1 / 10$ di quello di una torre circolare grande o della serie di Nardò.

\section{e. Torri a pianta ottagonale}

In provincia di Lecce è presente unicamente Torre S. Giovanni Marittimo ad Ugento, attualmente adibita a faro. Le restanti due, con pianta a stella, sono poste agli estremi di Terra d'Otranto (nelle province di Brindisi e di Taranto) e ricordano, in misura ridotta, le fortezze stellate del tardo rinascimento.

Le torri tipiche del Regno sono le più numerose. Esse sorgono spesso nelle zone periferiche dove l'iniziativa privata non aveva avuto stimoli; non a caso mancano del tutto nel Basso Salento, da Tricase a Gallipoli, dove già da tempo si era provveduto alla difesa anticorsara. Distinguibili come microsistemi a sè stanti sono le piccole torri della serie di Otranto, poste sugli speroni rocciosi nel luogo dello storico massacro, ma anche le grandi torri della serie di Nardò, testimonianze dell'importanza che quest'area ha avuto in passato. L'impianto delle torri anomale indica quasi sempre l'edificazione su iniziativa privata, precedentemente all'editto del 1563, poichè nate non da una progettazione generale alla base ma secondo le necessità e le possibilità di ciascun committente.
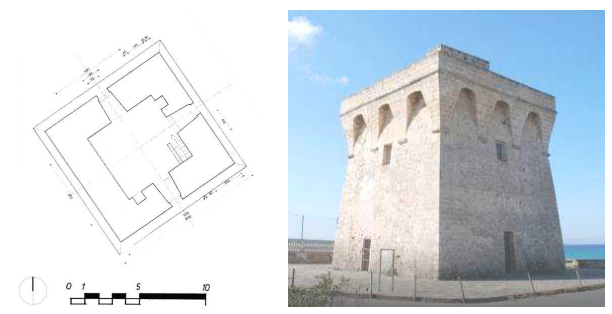

Figg. 2-3. Torre S. Giovanni la Pedata, tipologia "tipica del Regno", Gallipoli (LE).
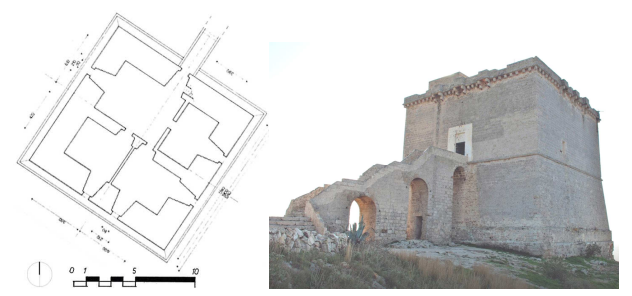

Figg. 4-5. Torre di S. Maria dell'Alto, tipologia "serie di Nardò", Nardò (LE).
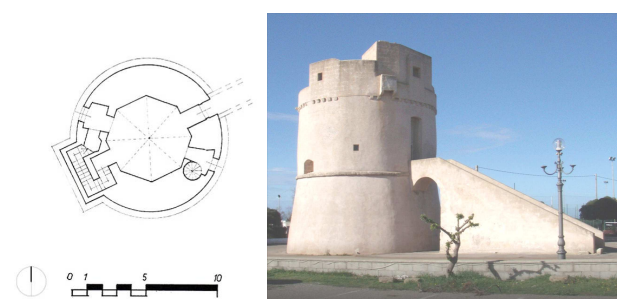

Figg. 6-7. Torre Suda, tipologia "rotonda media", S. Cesarea Terme (LE).
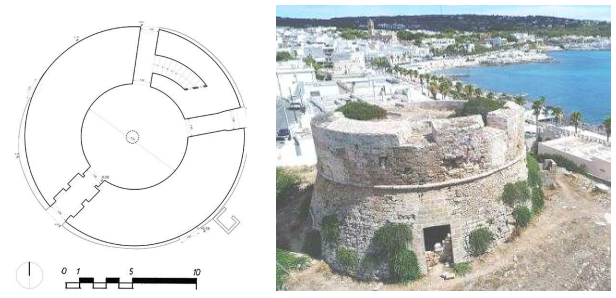

Figg. 8-9. Torre dell'Omomorto, tipologia "rotonda grande", Leuca (LE).
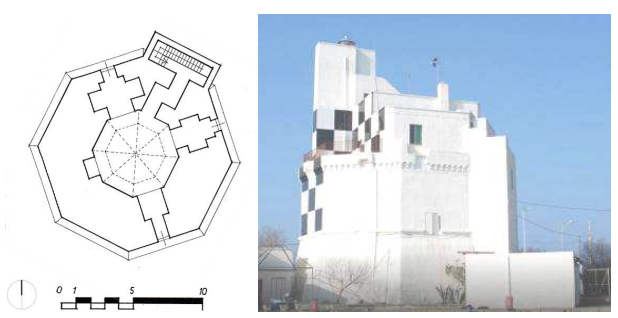

Figg. 10-11. Torre S. Giov. Marittimo, tipologia "ottagonale", Ugento (LE). 


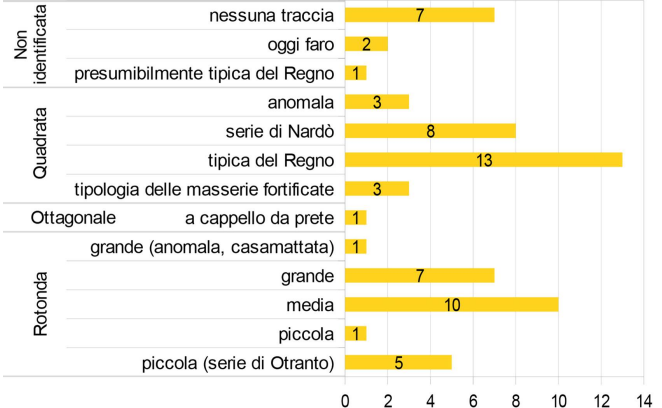

Fig. 12. Grafico indicante la concentrazione delle torri esaminate in funzione della loro tipologia.

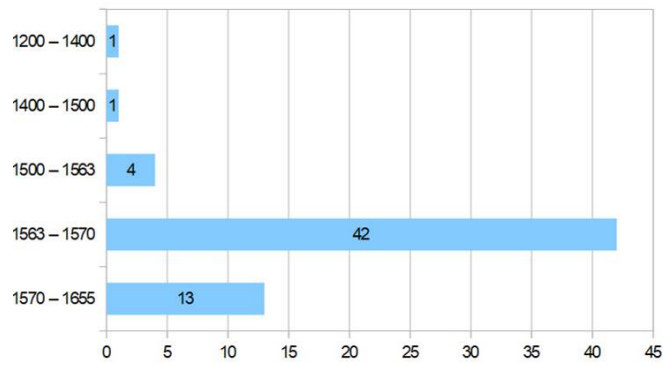

Fig. 13. Grafico indicante la concentrazione delle torri esaminate in funzione della loro periodizzazione cronologica.

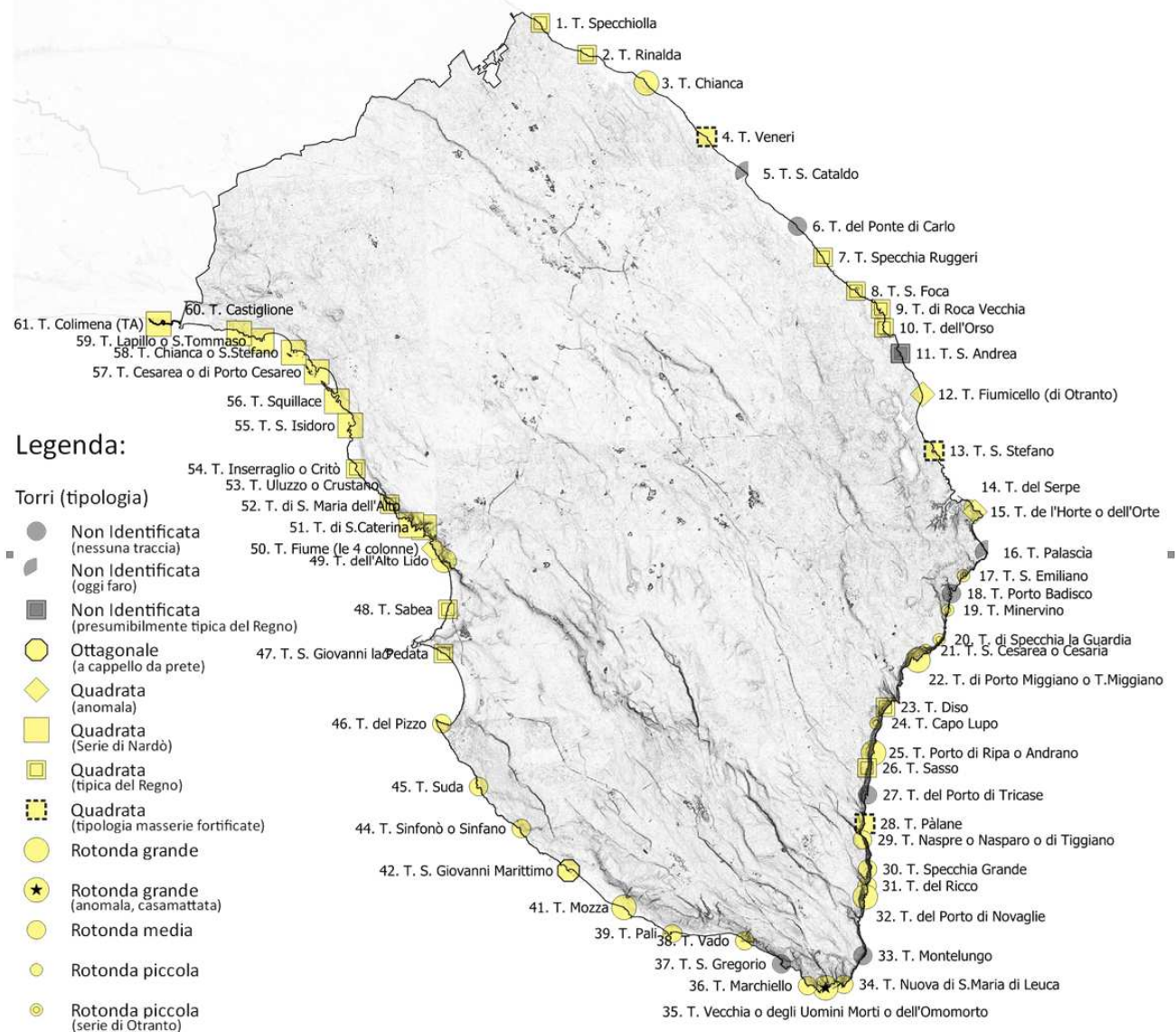

$\begin{array}{llllll}0 & 10 & 20 & 30 & 40 & 50 \mathrm{~km}\end{array}$

Fig. 14. Carta tematica indicante le torri costiere della provincia di Lecce in relazione alla loro tipologia. 


\subsection{Periodizzazioni cronologiche}

Le datazioni, desunte per lo più dal già citato censimento, sono state dedotte dallo studioso a seguito del confronto dei vari elenchi di torri redatti negli ultimi secoli.

La più antica è Torre del Serpe, probabilmente in origine un antico faro di età romana restaurato nel XIII secolo dall'imperatore svevo Federico II, seguita da Torre San Cataldo posta a difesa della marina di Lecce.

Antecedenti l'elenco del Vicerè sono ancora le torri adriatiche della serie di Otranto, quelle joniche della serie di Nardò e alcune torri costruite sull'estremo meridionale nei pressi del Capo di Leuca.

La maggior parte delle torri risulta edificata o ripristinata negli anni che seguono l'editto vicereale; "Il lungo elenco del Vicerè rappresenta tutte le torri agibili nel 1569 [...] e ha valore in negativo, cioè per le torri che non nomina, perchè è da presumere che sia stato stilato con ogni scrupolo di ben comparire, e che quindi le torri non elencate possano senz'altro considerarsi non ancora [o non più] agibili in quell'anno" (Faglia, 1978).

Le torri tarde testimoniano invece le difficoltà e quindi i ritardi avuti nella realizzazione del sistema difensivo litoraneo.

\subsection{Quote altimetriche}

A causa dell' assenza di importanti elevazioni del livello del suolo, quasi tutte le torri giacciono a pochi metri sul livello del mare.

Sulla parte orientale del Salento delle Serre, tra Otranto e Leuca, sono presenti le uniche torri edificate ad una quota rilevante, sugli strapiombi rocciosi delle terminazioni delle serre salentine.

In prossimità di Otranto vi è l'incrocio di due reti di avvistamento aventi funzioni complementari: "una alta sul costone roccioso per l'avvistamento lontano e la segnalazione ai paesi dell'entroterra, e una bassa sul mare per la segnalazione dei punti di sbarco" (Faglia, 1978).

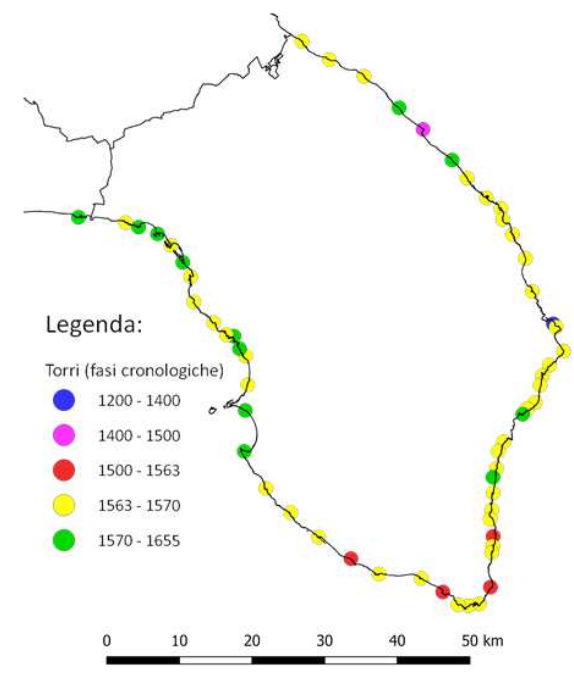

Fig. 15. Carta tematica indicante le torri costiere della provincia di Lecce in relazione alla loro periodizzazione.

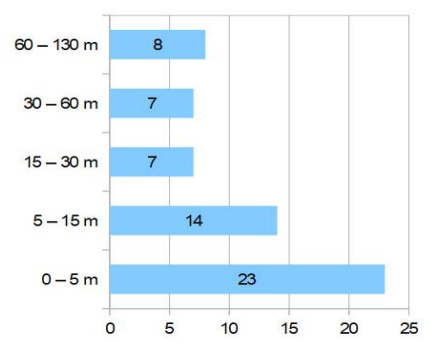

Fig. 16. Grafico indicante la concentrazione delle torri esaminate in funzione della loro quota s.l.m.

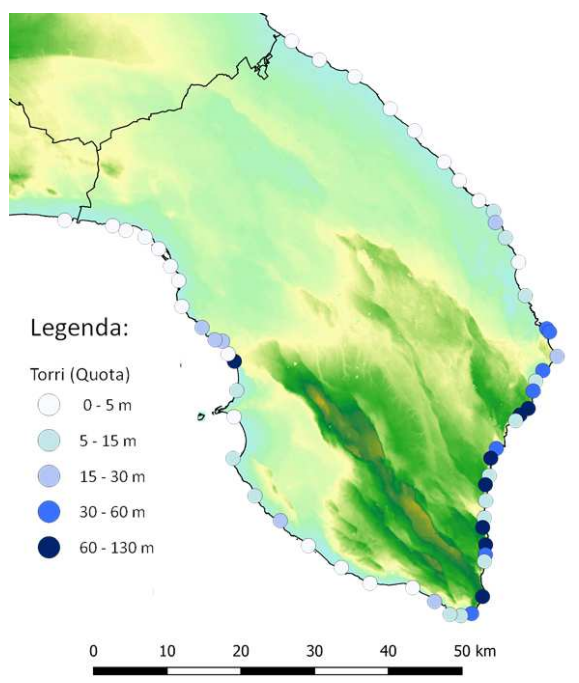

Fig. 17. Carta tematica indicante le torri costiere della provincia di Lecce in relazione alla loro quota s.1.m. 


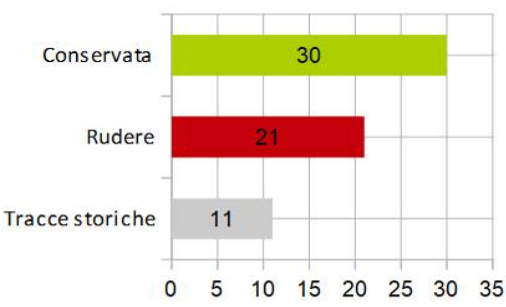

Fig. 18. Grafico indicante la concentrazione delle torri esaminate in funzione del loro stato di consistenza.

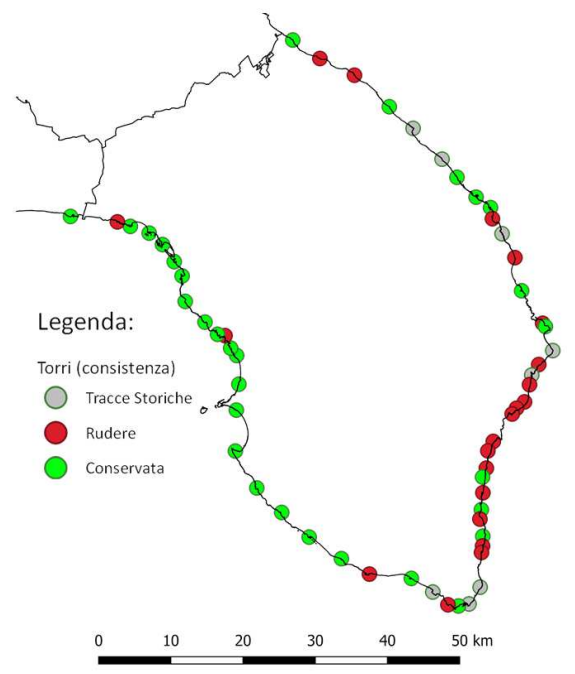

Fig. 39. Carta tematica indicante le torri costiere della provincia di Lecce in relazione al loro stato di consistenza.

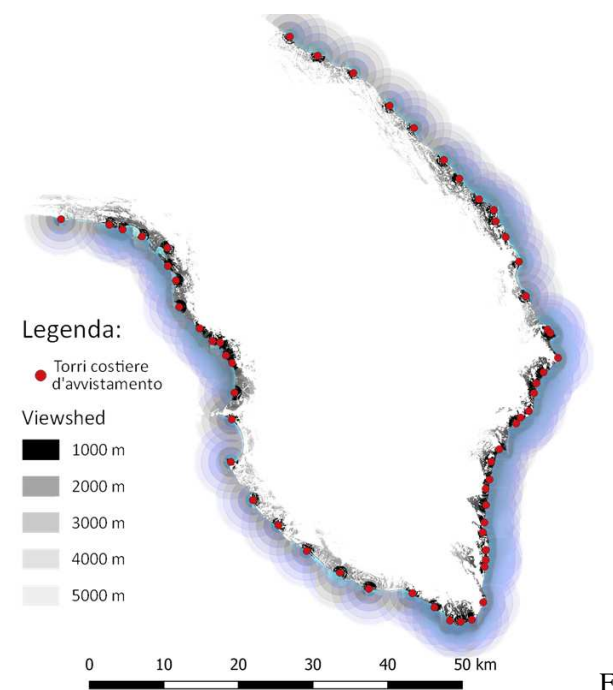

Fi

g. 20. Carta tematica illustrante il campo di visibilità delle torri costiere della provincia di Lecce in relazione all'orografia del territorio.

\subsection{Stato di consistenza}

Le torri attualmente riconoscibili in provincia di Lecce sono 49, tuttavia dalla documentazione storica degli elenchi redatti in epoche diverse se ne desume l'esistenza di altre 11 , portando così il numero ipotizzato complessivo a 60 torri.

Delle 49 ancora esistenti, 28 conservano ancora un'integrità strutturale mentre le rimanenti 21 versano in stato di rudere.

Le torri versanti in condizioni peggiori sono quelle della serie di Otranto poichè situate in luoghi impervi e poco atte ad un riutilizzo a causa della loro limitata dimensione.

Le meglio tenute, oltre a quelle restaurate negli scorsi decenni che ora hanno una utilizzazione, sono le torri della serie di Nardò poichè di esse si ha avuto un utilizzo continuativo con funzione di tutela dei campi agricoli.

\subsection{Analisi di visibilità}

Per rendere funzionante il sistema costiero di torri occorreva che tutte includessero nel loro raggio visuale anche le due torri contigue. E' questo, infatti, il motivo che spinse nel 1563 gli ingegneri militari al servizio del Vicerè ad ispezionare le coste del Regno per apprendere dove meglio edificare le nuove torri, al fine di collegarle visivamente con quelle già esistenti in un unico, grande e continuo sistema territoriale di difesa incardinato sulle torri d'avvistamento.

In seguito alla realizzazione di questo grandioso progetto, le distanze tra due torri contigue rientreranno quasi sempre tra i 2 e i $5 \mathrm{~km}$, garantendo un'efficace copertura visiva della linea di costa.

\section{Conclusioni}

Ancora oggi, percorrendo la costa pugliese, è possibile scorgere le innumerevoli torri che la puntellano solitarie, per la maggior parte prive di un sistema organizzativo che le includa in un qualche circuito culturale, risultando sconosciute ai più e in prevalenza abbandonate. E' sulla base di queste premesse che si considera essenziale ri-conoscerle, restituendo loro una dignità storica e testimoniale dell'identità di quei luoghi che per 
secoli sono stati connotati dalla loro unica presenza. Lo studio, realizzato in forma quanto più integrale e rigorosa, esprime la volontà di comprensione dei diversi aspetti storici, costruttivi e simbolici che furono alla base della loro edificazione e, seguendo una metodologia fondata su una dialettica tra teoria e prassi, conoscenza ed intervento, si propone come esempio di metodo propedeutico ad un progetto di restauro e valorizzazione integrata.

\section{Note}

Il contributo si basa sul lavoro svolto nella tesi di Laurea Magistrale (Tricarico, 2019). Grafici e cartografie sono di produzione propria.
${ }^{1}$ Tra le torri esaminate è stata inclusa anche Torre Colimena, sita ad Avetrana (TA), considerata in analogia con le torri leccesi della serie di Nardò.

2 Per una esaustiva classificazione tipologica delle torri ci si è affidati al lavoro svolto da Faglia, et al., 1978.

3 Torre Castiglione, indicata dal Faglia come della serie di Nardò, è qui rappresentata come tipica del Regno seguendo i più aggiornati e approfonditi studi di F. Errico che la accosta "con chiarezza, e in maniera inequivocabile [...] alla serie delle torri "tipiche del Regno", contrariamente a quanto ipotizzato da Vittorio Faglia (1978)" (Errico, 2018).

\section{Bibliography}

Brunetti, O. (2006). A difesa dell'impero: pratica architettonica e dibattito teorico nel Viceregno di Napoli nel Cinquecento, Congedo Editore, Galatina.

Cancila, R. (2007). Mediterraneo in armi (secc. XV-XVIII), Associazione Mediterranea, Palermo.

Cosi, G. (2006). Cronache del Cinquecento salentino (da documenti notarili), Pubbligraf, Alessano.

Errico, F. (2018). "Le torri della «serie di Nardò»: caratteri morfologici e analisi comparative", in RodrìguezNavarro, P.; Marotta, A.; Spallone, R., eds., FORTMED 2018. Defensive architecture of the Mediterranean, XV to XVIII centuries: proceedings of the International conference on Modern age Fortification of the Mediterranean coast, Politecnico di Torino, vol. VIII, pp. 587-593.

Faglia, V.; Bruno, F.; Losso, G.; Manuele, A. (1978). Censimento delle torri costiere nella provincia di Terra d'Otranto: indagine per il ricupero nel territorio. Il restauro di Torre Sabea a Gallipoli, Istituto italiano dei castelli, Roma.

Giorgi, C. De. (1981). Note e ricerche sui materiali edilizi adoperati nella provincia di Lecce, Congedo Editore, Galatina.

Mutalipassi, S. (2006). Un sistema di fortificazioni nel Salernitano: le torri costiere della piana del Sele; conoscenza e indirizzi di conservazione, Tesi di dottorato, Università degli Studi di Napoli l'Orientale, Napoli.

Pace, G. (1992). "Itinerari culturali per una «conservazione integrata» delle fortificazioni”, in Notarangelo, A. De., Torri e castelli nel Mezzogiorno. Recupero, territorio, innovazione, integrazione, Giannini, Napoli.

Petrignani, A. (2001). "Aspetti funzionali e urbanistici dell'architettura militare”, in Vita, R. De, Castelli, torri ed opere fortificate di Puglia, M. Adda Editore, Bari, pp. 377-392.

Tricarico, G. (2019). Il sistema pugliese di fortificazioni costiere: conoscenza, conservazione e valorizzazione della Torre dell'Omomorto in Santa Maria di Leuca (LE), Tesi di L.M. In Architettura per il Progetto Sostenibile, Politecnico di Torino, Torino.

Vita, R. De. (2001). Castelli, torri ed opere fortificate di Puglia, M. Adda Editore, Bari. 